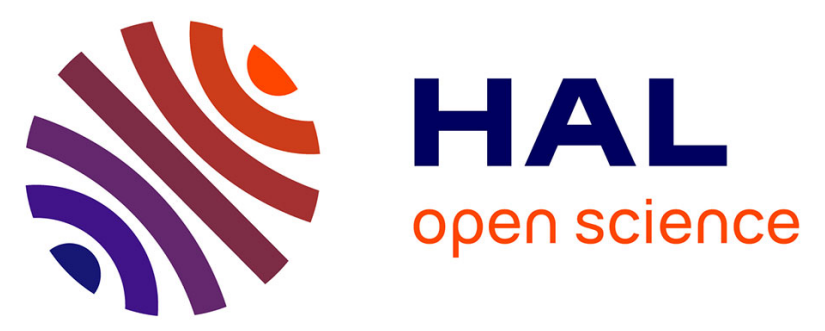

\title{
Allelic composition of MdMYB1 drives red skin color intensity in apple (Malus x domestica Borkh.) and its application to breeding
}

Shigeki Moriya, Miyuki Kunihisa, Kazuma Okada, Taku Shimizu, Chikako Honda, Toshiya Yamamoto, Helene Muranty, Caroline Denance, Yuichi Katayose, Hiroyoshi Iwata, et al.

\section{To cite this version:}

Shigeki Moriya, Miyuki Kunihisa, Kazuma Okada, Taku Shimizu, Chikako Honda, et al.. Allelic composition of MdMYB1 drives red skin color intensity in apple (Malus x domestica Borkh.) and its application to breeding. Euphytica, 2017, 213 (4), pp.1-13. 10.1007/s10681-017-1864-x . hal01603832

\section{HAL Id: hal-01603832 \\ https://hal.science/hal-01603832}

Submitted on 26 May 2020

HAL is a multi-disciplinary open access archive for the deposit and dissemination of scientific research documents, whether they are published or not. The documents may come from teaching and research institutions in France or abroad, or from public or private research centers.
L'archive ouverte pluridisciplinaire HAL, est destinée au dépôt et à la diffusion de documents scientifiques de niveau recherche, publiés ou non, émanant des établissements d'enseignement et de recherche français ou étrangers, des laboratoires publics ou privés. 


\title{
Allelic composition of $M d M Y B 1$ drives red skin color intensity in apple (Malus $\times$ domestica Borkh.) and its application to breeding
}

\author{
Shigeki Moriya $\cdot$ Miyuki Kunihisa $\cdot$ Kazuma Okada $\cdot$ Taku Shimizu $\cdot$ Chikako Honda • \\ Toshiya Yamamoto $\cdot$ Hélène Muranty $\cdot$ Caroline Denancé $\cdot$ Yuichi Katayose • \\ Hiroyoshi Iwata $\cdot$ Kazuyuki Abe
}

Received: 25 November 2016/ Accepted: 25 February 2017/Published online: 8 March 2017

(C) Springer Science+Business Media Dordrecht 2017

\begin{abstract}
Since apple fruit skin reddens poorly under warmer climates, new apple cultivars are desired that are adapted to global warming in terms of bearing well-reddened fruit. We developed a simple sequence repeat marker, Mdo.chr9.4, which is suitable for red skin color selection. It amplified four alleles (Mdo.chr9.4- $\mathrm{R}_{0}$, Mdo.chr9.4-Y ${ }_{-3}$, Mdo.chr9.4$\mathrm{Y}_{-9}$, and Mdo.chr9.4-Y $\mathrm{Y}_{-15}$ ) distinguished by length. Mdo.chr9.4- $\mathrm{R}_{0}$ associated with $M d M Y B 1-1$ which confers red fruit skin. The presence of Mdo.chr9.4- $\mathrm{R}_{0}$ was consistent with empirical skin color in all 160 tested accessions. Mdo.chr9.4 was identified as the only
\end{abstract}

Electronic supplementary material The online version of this article (doi:10.1007/s10681-017-1864-x) contains supplementary material, which is available to authorized users.

S. Moriya $(\bowtie) \cdot$ K. Okada $\cdot$ T. Shimizu

C. Honda $\cdot$ K. Abe

Apple Research Station, Institute of Fruit Tree and Tea

Science, National Agriculture and Food Research

Organization (NARO), 92-24 Shimokuriyagawa

Nabeyashiki, Morioka, Iwate 020-0123, Japan

e-mail: moriyas@affrc.go.jp

M. Kunihisa · T. Yamamoto

Institute of Fruit Tree and Tea Science, NARO, 2-1

Fujimoto, Tsukuba, Ibaraki 305-8604, Japan

H. Muranty · C. Denancé

IRHS, INRA, AGROCAMPUS-Ouest, Université

d'Angers, SFR 4207 QUASAV, Université Bretagne

Loire, 42 rue Georges Morel BP60057, Beaucouzé 49071,

France significant marker that contributed to red skin color intensity by a genome wide association study (GWAS), and it accounted for $52.0 \%$ of phenotypic variation, confirming that $M d M Y B 1$ was the major and principal determinant of fruit skin color in apples. Individuals with a homozygous state of Mdo.chr9.4- $\mathrm{R}_{0}$ (dose 2) were significantly redder than those showing a heterozygote state (dose 1) in both the accession set and full-sib families, indicating a partially dominant effect of MdMYB1-1. Therefore, the selection of dose 2 individuals would target individuals with intensive red skin. We applied Mdo.chr9.4 to several application populations using a time and cost-efficient genotyping system developed in the present study. This system, along with Mdo.chr9.4, provide advanced marker-assisted breeding

\footnotetext{
Y. Katayose

Advanced Genomics Breeding Section, Institute of Crop

Science, NARO, 1-2 Ohwashi, Tsukuba,

Ibaraki 305-8634, Japan

H. Iwata

Department of Agricultural and Environmental Biology, Graduate School of Agricultural and Life Sciences, The

University of Tokyo, 1-1-1 Yayoi, Bunkyo-ku,

Tokyo 113-8657, Japan
} 
for intensive red skin color apples adapted to a global warming climate.

Keywords Anthocyanin - Fruit color - Genomewide association study · Marker-assisted selection . Quantitative trait

\section{Introduction}

The red skin color of apples is one of the major determinants of consumer preference; generally, people prefer intensive and broadly-covered red skin. Such fruit is traded at a higher price than poor-colored fruit as a reflection of market acceptance. Therefore, achieving good coloration on fruit skin is a major interest for both fruit producers and markets. However, recently, in the context of global warming, high temperature causes poor coloration, especially on early harvested cultivars (Lin-Wang et al. 2011). Possible solutions may include treatments for skin coloration (Ju et al. 1999; Iglesias et al. 2005) and replanting orchards with new cultivars whose fruits are intensive red under warmer climates. Therefore, it is desirable to develop new apple cultivars that are adapted to a warm climate in terms of bearing fruit with an intensive red skin.

The red coloration in apple skin is mainly due to an accumulation of anthocyanin. It is controlled by two categories of genes, including enzymatic genes such as structural biosynthetic genes and regulatory genes such as transcription factors. Among them, MdMYBI/ $A / 10$, an R2R3 MYB transcription factor, has been reported to be a key regulator (Takos et al. 2006; Ban et al. 2007; Espley et al. 2007). An allele specific PCR concluded that these three genes are allelic (Lin-Wang et al. 2010). Thus, from this point forward $M d M Y B 1$ is used as their representative name in this study.

There are at least four alleles in MdMYBI (Takos et al. 2006). Three of them (MdMYB1-1, MdMYB1-2, and $M d M Y B 1-3$ ), called $\mathrm{R} 1$ types, play a role in fruit skin coloration, whereas the rest ( $M d M Y B 10$ ), called R6 type, confers red flesh along with almost the entire tree, such as fruit skin and foliage (Espley et al. 2009). Among the R1 type alleles, $M d M Y B 1-1$ is the only allele associated with the red skin color phenotype. MdMYB1-1 behaves as a dominant factor for fruit skin color; thus, individuals that are heterozygous (dose 1) or homozygous (dose 2) of MdMYB1-1 bear red skinned fruit, whereas those that are a null state (dose 0 ) bear yellow or sometimes green skin. Despite the fact that there are no functional differences among the MdMYB1-1, MdMYB1-2, and MdMYB1-3 translation products, the expressions of MdMYB1-2 and MdMYB1-3 are so little that they confer a yellow skin (Takos et al. 2006).

To date, there have been several DNA markers that discriminate MdMYB1-1 from MdMYB1-2 to MdMYB1-3 (Takos et al. 2006; Yuan et al. 2014; Zhang et al. 2014). However, there were inconsistencies between genotype and phenotype, even in major well-known green cultivars such as 'Granny Smith'. Therefore, new DNA markers that correctly distinguish $M d M Y B 1$ alleles are desired.

By applying the dominant model described above, distinguishing between MdMYB1-1 dose 1 and 2 individuals from dose 0 can achieve a marker-assisted selection (MAS) for skin color to obtain a red-skinned apple in breeding. However, an application in a practical breeding population found individuals that had inconsistent genotype and phenotype (Zhu et al. 2011). Moreover, in addition to the selection of such individuals, selection of intensive red-skinned individuals is of interest because they are considered to adapt well to a warm climate. While a genome wide association (GWA) approach has already been attempted for fruit color in apples (Kumar et al. 2013; Lozano et al. 2014; Migicovsky et al. 2016), they did not focus on skin color intensity in detail. Therefore, the identification of the genetic factors that control skin color intensity is of interest to achieve an advanced MAS for skin color.

To apply MAS in practical breeding, several trials to optimize various protocol steps have been performed, including seedling nursing, DNA extraction, and marker detection (Baumgartner et al. 2016; EdgeGarza et al. 2014; Chagné et al. 2016). However, the proposed MAS protocols need transplanting before genotyping (Baumgartner et al. 2016; Edge-Garza et al. 2014) and use harmful reagents such as liquid nitrogen (Edge-Garza et al. 2014). Therefore, they should be further improved for their efficiency and be optimized in our breeding scheme.

The aim of our study was to develop a MAS system to select individuals that had an intensive red skin color. We tried to identify the genetic factors that control skin color using the GWA approach and developed a DNA marker that could be routinely used in MAS. Lastly, we developed a high-throughput and cost-efficient genotyping system and applied it to our breeding program. 


\section{Materials and methods}

Plant materials and DNA extraction

One hundred sixty apple accessions, hereafter the accession set (AS), were used for genome wide association study (GWAS; Table 1; Online Resource 1). Parental information of AS is available in Kunihisa et al. (2016). Five full-sib families (FSs) composed of 218 individuals in addition to AS were used for the dose effect validation (Table 2). Trees were planted in the orchard of the Apple Research Station, Institute of Fruits Tree and Tea Science, National Agriculture and Food Research Organization, Japan and grafted on dwarfing rootstocks e.g., JM1, JM7, JM8, M.9A, M.9 EMLA, M.26, and M.26 EMLA. They were treated and pruned the same way as in commercial fruit production in Japan. Genomic DNA was extracted by a DNeasy Plant Mini Kit (Qiagen, Hilden, Germany) or automated DNA extraction machine PI-50 $\alpha$ (Kurabo, Osaka, Japan).

Five application families (AFs) composed of 872 individuals were used for obtaining a proof of concept of the genotyping system (Table 3). These crosses were made in May. Obtained seeds were sown in Jiffy 7 peat pellet (Jiffy International AS., Kristiansand, Norway) in a greenhouse the following March. They were placed in an 8 lines $\times 12$ rows format in plastic trays in order to imitate the format of a 96-well PCR plate. Genomic DNA was extracted from seedlings at the 1-3 leaf stage by a simple method described by Thompson and Henry
(1995), with some modifications. Namely, approximately a $2 \mathrm{~mm}^{2}$ area of young leaf tips were collected and placed in the corresponding wells of a 96-well PCR plate. Collected leaves were stored at $-80{ }^{\circ} \mathrm{C}$ until use. Then, $25 \mu \mathrm{l}$ of a template preparation solution [100 mM Tris-HCl (pH 9.5), $1 \mathrm{M} \mathrm{KCl,} 10$ mM EDTA (pH 8.0)] was added to each well followed by heating to $95^{\circ} \mathrm{C}$ for $10 \mathrm{~min}$. Samples were diluted with $100 \mu \mathrm{l}$ distillated water and a $1 \mu \mathrm{l}$ of diluted solution was immediately used as PCR templates.

Fruit color evaluation

The red skin color was evaluated for at least one and at most 25 years depending on the genotype. For evaluation, two well-colored fruits per genotype were harvested when the fruit ground color at the calyx end turned to a yellowish-green or yellow, as described by Kunihisa et al. (2014). Then, the fruit over color (OC) and percent of fruit surface that was red (i.e., percent over color; POC) were evaluated by an ordinal scale (1-5; Online Resource 2), which gave an average score for one genotype. The year effect was calculated by the "effect" command in the R package "Effect," then the adjusted mean was calculated from adjusted yearly data. Because of this adjustment, some data points exceeded the value 5. The overall color intensity (OCI) assumed that the total amount of anthocyanin in fruit skin was determined by the following formula,

Table 1 Genotypes of the simple sequence repeat marker Mdo.chr9.4 and skin color phenotype in representative accessions

\begin{tabular}{|c|c|c|c|c|c|c|}
\hline Accession & Genotype of Mdo.chr9.4 $4^{\mathrm{a}}$ & Phenotype $^{c}$ & Overcolor & Percent overcolor & Over color intensity $^{\mathrm{b}}$ & Years tested \\
\hline Jonathan & $\mathrm{R}_{0} / \mathrm{R}_{0}$ & Red & 4.57 & 4.09 & 18.69 & 9 \\
\hline Ralls Janet & $\mathrm{R}_{0} / \mathrm{R}_{0}$ & Red & 3.18 & 3.60 & 11.43 & 5 \\
\hline Gala & $\mathrm{R}_{0} / \mathrm{Y}_{-3}$ & Red & 3.30 & 3.27 & 10.77 & 3 \\
\hline Starking delicious & $\mathrm{R}_{0} / \mathrm{Y}_{-15}$ & Red & 4.36 & 4.90 & 21.36 & 4 \\
\hline Fuji & $\mathrm{R}_{0} / \mathrm{Y}_{-15}$ & Red & 2.95 & 4.20 & 12.37 & 25 \\
\hline Indo & $\mathrm{Y}_{-3} / \mathrm{Y}_{-3}$ & Green & 1.97 & 1.56 & 3.07 & 7 \\
\hline Golden delicious & $\mathrm{Y}_{-3} / \mathrm{Y}_{-15}$ & Yellow & 1.03 & 1.08 & 1.11 & 7 \\
\hline Granny Smith & $\mathrm{Y}_{-3} / \mathrm{Y}_{-9}$ & Green & 1.02 & 0.92 & 0.93 & 5 \\
\hline
\end{tabular}

The full list of genotypic/phenotypic data is shown in the Online Resource 1

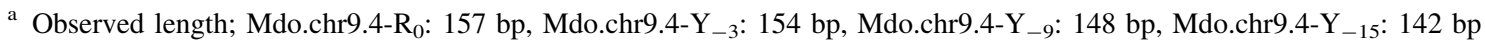

b Over color intensity was determined by the over color score multiplied by the percent over color score

c Photographs of these cultivar phenotypes are provided in Online Resource 4 
Table 2 Overall color intensity difference based on the dose of Mdo.chr9.4- $\mathrm{R}_{0}$ in full-sib families

\begin{tabular}{|c|c|c|c|c|c|c|}
\hline \multirow[t]{2}{*}{ Populations } & \multirow[t]{2}{*}{ Dose of Mdo.chr9.4- $\mathrm{R}_{0}$} & \multirow[t]{2}{*}{ No. of plants } & \multicolumn{4}{|c|}{ Over color intensity } \\
\hline & & & Mean & & Maximum & Minimum \\
\hline \multirow[t]{3}{*}{ Fuji $\times$ Hatsuaki } & 0 & 19 & 3.34 & $\mathrm{a}^{\mathrm{a}}$ & 14.74 & 1.18 \\
\hline & 1 & 30 & 13.62 & $\mathrm{~b}$ & 17.81 & 2.07 \\
\hline & 2 & 27 & 14.92 & $\mathrm{~b}$ & 27.25 & 9.48 \\
\hline \multirow[t]{3}{*}{ Fuji $\times$ Tsugaru } & 0 & 8 & 2.08 & $\mathrm{a}$ & 5.23 & 0.71 \\
\hline & 1 & 21 & 11.90 & $\mathrm{~b}$ & 16.45 & 4.93 \\
\hline & 2 & 6 & 14.75 & $\mathrm{~b}$ & 23.45 & 8.08 \\
\hline \multirow[t]{2}{*}{ Fuji $\times$ Kotaro } & 1 & 13 & 13.25 & $\mathrm{a}$ & 16.92 & 9.48 \\
\hline & 2 & 6 & 15.95 & $\mathrm{a}$ & 21.45 & 13.14 \\
\hline \multirow[t]{2}{*}{ Hatsuaki $\times$ Megumi } & 1 & 30 & 12.74 & $\mathrm{a}$ & 24.08 & 1.49 \\
\hline & 2 & 27 & 16.22 & $\mathrm{~b}$ & 27.25 & 8.45 \\
\hline \multirow[t]{2}{*}{ Sensyu $\times$ Kotaro } & 1 & 19 & 12.61 & $\mathrm{a}$ & 17.81 & 1.49 \\
\hline & 2 & 12 & 16.49 & $\mathrm{~b}$ & 27.25 & 13.65 \\
\hline
\end{tabular}

${ }^{a}$ Different letters indicate significant differences at $P<0.05$, determined by Steel-Dwass tests or Kruskal-Wallis tests

Table 3 Marker-assisted selection for skin color intensity using a simple sequence repeat marker Mdo.chr9.4

\begin{tabular}{|c|c|c|c|c|c|c|}
\hline \multirow[t]{2}{*}{ Cross } & \multirow[t]{2}{*}{ Cross type } & \multicolumn{3}{|c|}{ Mdo.chr9.4- $\mathrm{R}_{0}$} & \multirow[t]{2}{*}{ Total } & \multirow[t]{2}{*}{$\chi^{2 a}$} \\
\hline & & Dose 2 & Dose 1 & Dose 0 & & \\
\hline Kotaro $\times$ Aikanokaori & $\mathrm{R}_{0} / \mathrm{R}_{0} \times \mathrm{R}_{0} / \mathrm{Y}_{-3}$ & 70 & 65 & & 135 & 0.18 \\
\hline Morioka $58 \times$ Slim Red & $\mathrm{R}_{0} / \mathrm{Y}_{-3} \times \mathrm{R}_{0} / \mathrm{Y}_{-15}$ & 18 & 98 & 37 & 153 & $16.8 * * *$ \\
\hline Haruka $\times$ Aikanokaori & $\mathrm{Y}_{-3} / \mathrm{Y}_{-15} \times \mathrm{R}_{0} / \mathrm{Y}_{-3}$ & & 102 & 132 & 234 & $3.85^{*}$ \\
\hline Shinano Gold $\times$ Aikanokaori & $\mathrm{Y}_{-3} / \mathrm{Y}_{-15} \times \mathrm{R}_{0} / \mathrm{Y}_{-3}$ & & 114 & 80 & 194 & $5.96^{*}$ \\
\hline \multirow[t]{2}{*}{ Aikanokaori $\times$ Morioka 71} & $\mathrm{R}_{0} / \mathrm{Y}_{-3} \times \mathrm{Y}_{-3} / \mathrm{Y}_{-15}$ & & 82 & 74 & 156 & 0.41 \\
\hline & Total & 88 & 461 & 323 & 872 & \\
\hline
\end{tabular}

${ }^{a}$ Fitness to the theoretical segregation proportion by $\chi^{2}$ tests

* Significant at the 0.05 probability level

*** Significant at the 0.0001 probability level

$O C I_{i}=O C_{i} \times P O C_{i}$

where $\mathrm{OCI}_{\mathrm{i}}, \mathrm{OC}_{\mathrm{i}}$ and $\mathrm{POC}_{\mathrm{i}}$ are fruit coloration scores of ith genotype. We considered OCI as an index of ability of red coloration in this study.

\section{Development of a DNA marker flanking $M d M Y B 1$}

The sequence of contig MDC013323.319 (chr9:29,465, $538.29,488,265$ according to the apple genome version 1; Velasco et al. 2010) on which MdMYB1 (MDP0000259614; chr9:29,471,894.29,475,878) was located, was downloaded from the genome database for Rosaceae (GDR; Jung et al. 2014) and was subjected to simple sequence repeat (SSR) search by BatchPrimer3 (You et al. 2008). Primer sequences were designed from the flanking sequence of $\mathrm{AAT}_{7}+\mathrm{AAT}_{4}$ repeats located $12.3 \mathrm{kbp}$ downstream from the transcription start site of $M d M Y B 1$ as Mdo.chr9.4 (Mdo.chr9.4-F:TGTTGAGTTTATGATGAATATTTTGAA, Mdo.chr9.4-R gtttcttGCTTCCA GAAGTAAGACTTAAATCCA. Small cases indicate a pigtail sequence). Amplification of this marker was performed according to Moriya et al. (2012) and was detected with an automated sequencer CEQ8000 (Beckman Coulter). Its genomic position was investigated by the linkage mapping approach using a 
segregating population composed of $137 \mathrm{~F}_{1}$ individuals derived from 'Orin' $\times$ 'Akane' (Kunihisa et al. 2014).

\section{Apple 20K array detection and GWAS}

AS was genotyped using the $20 \mathrm{~K}$ single nucleotide polymorphism (SNP) array for apples (Bianco et al. 2014). Data trimming and imputation were performed as described by Moriya et al. (2016). Finally, a dataset of 16,010 SNPs was used for downstream analyses. The markers were aligned according to the apple genome version 2 available from the array information (Bianco et al. 2014) when we visualized the results. Markers aligned to only "scaffold" in the array information and thus lacking positional information were tentatively placed to a fictive chromosome, named chromosome "unanchored".

A linkage disequilibrium (LD) estimation was performed as described by Moriya et al. (2016). Calculation of $r^{2}$ was performed using only those markers that showed a minor allele frequency of more than 0.05 .

The GWA analysis was performed using a mixed model (Yu et al. 2006) in MLMM software (Segura et al. 2012) implemented in $R$ ( $R$ Core Team 2016). We included Mdo.chr9.4 into the GWAS scored as the dose of the Mdo.chr9.4- $\mathrm{R}_{0}$ allele (see Results for allele naming). We placed Mdo.chr9.4 at the end of unanchored chromosome. To investigate the appropriate number of principal components (PCs) to include in the GWAS in order to control the population structure, a model selection analysis using GAPIT software (Lipka et al. 2012) was performed. To select the best number of SNP cofactors in the MLMM, extended Bayesian information content (eBIC) was applied. The proportion of variation explained by SNP, kinship, and residual was obtained by the "mlmm" command in MLMM software.

\section{Statistics}

All statistical procedures were conducted with $\mathrm{R}$ version 3.3.0. For the Steel-Dwass test, we used a publicly available function on a web page of Gunma University, Japan (http://aoki2.si.gunma-u.ac.jp/R/ Steel-Dwass.html; accessed 2 September, 2016).
The quantitative effect of $M d M Y B 1-1$ was calculated using mean $\mathrm{OCI}$ of Mdo.chr9.4- $\mathrm{R}_{0}$ doses as an index. The calculation was as follows:

$\mathrm{A}=\left(\mathrm{OCI}_{\text {dose } 2}-\mathrm{OCI}_{\text {dose } 1}\right) / 2$

and

$\mathrm{D}=\left(\mathrm{OCI}_{\mathrm{dose} 1}-\mathrm{OCI}_{\mathrm{dose} 0}\right)-\mathrm{A}$

then, the level of dominance was determined by looking at $\mathrm{D} / \mathrm{A}$. $\mathrm{D} / \mathrm{A}=1$ : complete dominance, $0<\mathrm{D} / \mathrm{A}<1$ : incomplete dominance, $\mathrm{D} / \mathrm{A}>1$ : overdominance.

\section{Results}

Characteristics of Mdo.chr9.4

Mdo.chr9.4 showed polymorphism in the alleles of 'Orin' and was mapped at the bottom of linkage group 9 where we designed this marker (Online Resource 3). Thus, it was confirmed that Mdo.chr9.4 amplified a sequence close to MdMYB1. Mdo.chr9.4 amplified four alleles distinguished by length. In major cultivars and founders, the red skin color phenotype was only associated with the $157 \mathrm{bp}$ allele designed as Mdo.chr9.4- $\mathrm{R}_{0}$ (Table 1; Online Resource 4). Therefore, Mdo.chr9.4- $\mathrm{R}_{0}$ was associated with MdMYB1-1 that confers red coloration in fruit skin. We then designated the other alleles as follows: amplified alleles of 154, 148, and $142 \mathrm{bp}$ were named Mdo.chr9.4-Y ${ }_{-3}$, Mdo.chr9.4-Y_9, and Mdo.chr9.4$\mathrm{Y}_{-15}$, respectively. These three alleles were associated with yellow skin color, and shorter by 3, 9, and $15 \mathrm{bp}$ than Mdo.chr9.4- $\mathrm{R}_{0}$, respectively. The Mdo.chr9.4$\mathrm{Y}_{-9}$ allele was observed only in 'Granny Smith'. The full list of genotypes is available in Online Resource 1.

Phenotypic assessment

The mean tested years for one genotype was 7.14 in AS (Online Resource 1), whereas it was 1.34 in FS (data not shown). The distribution of OCIs in the two datasets showed two peaks, one corresponding to nonred fruit-skinned individuals and the other to red fruitskinned individuals (Fig. 1; Online Resource 1). The first peak consisted of individuals with dose 0 of Mdo.chr9.4- $\mathrm{R}_{0}$, whereas the other consisted of those 

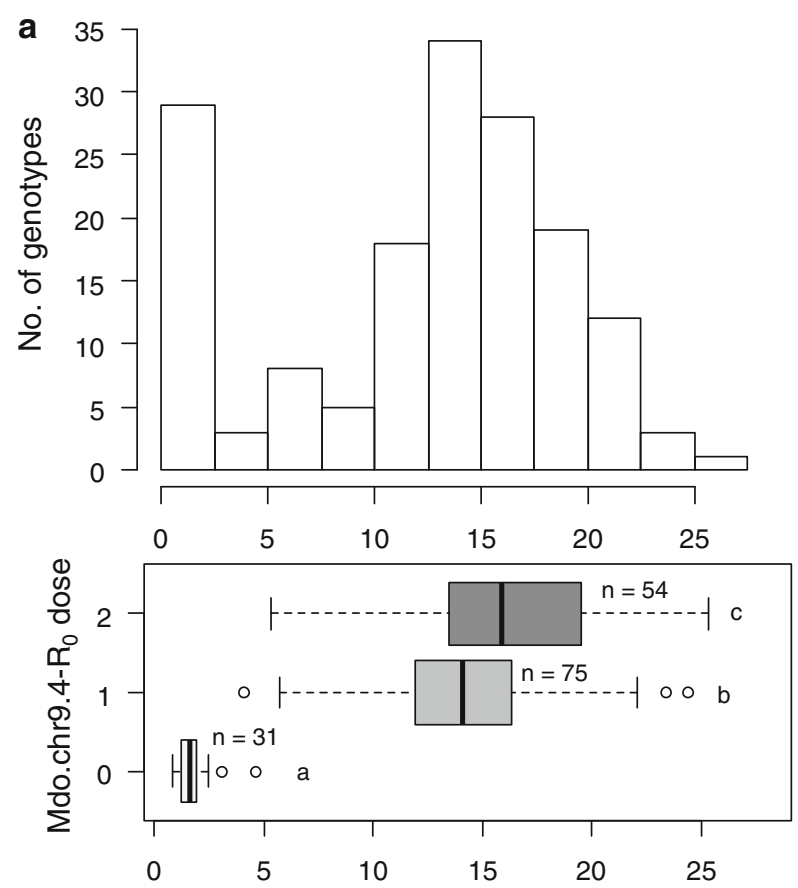

b
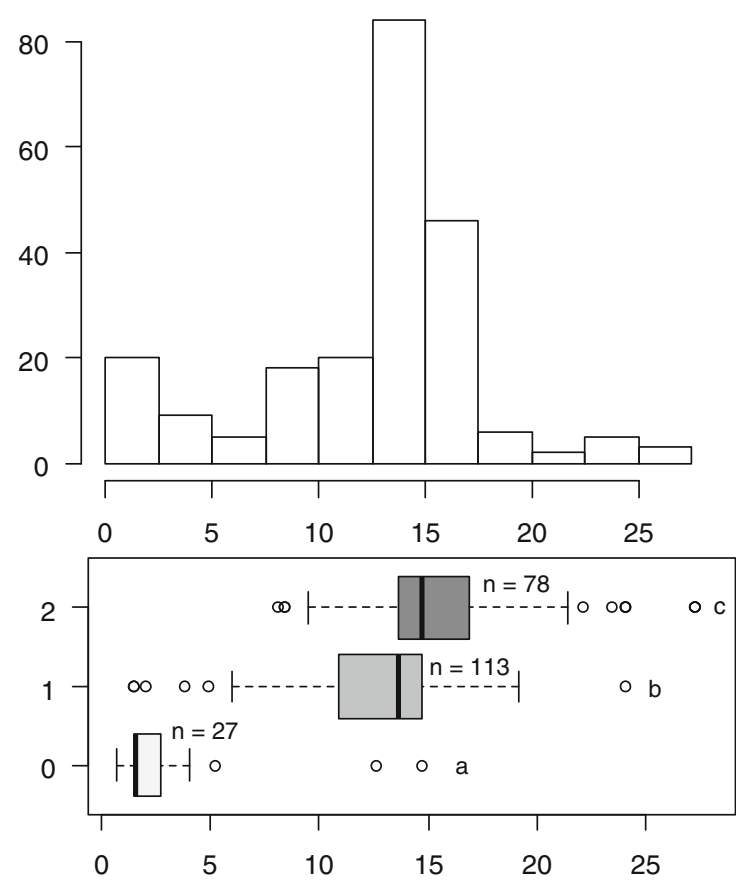

Overall color intensity

Fig. 1 Distribution of overall color intensity (OCI) in the accession set (a) and full-sib families (b). Histograms of the OCI distribution are shown in the upper panels. Box plots of the OCI based on dose of Mdo.chr9.4- $\mathrm{R}_{0}$ are shown in the lower panels.
The number of genotypes within doses are shown beside the boxes in the lower panels. Different letters indicate significant differences at $P<0.05$, as determined by Steel-Dwass tests

structure in apple. At first, the SNP RosBREEDSN P_SNP_GT_33056483_Lg9_01200_MAF50_MDP00 00254312_exon19 (rs267612505, at chr9:29,696kbp, according to the apple genome version 1) was identified as the most significant marker (data not shown). However, this SNP showed only two classes, i.e., only $\mathrm{AA}$ and $\mathrm{AB}$ genotypes existed. The comparison of genotypes between this SNP and Mdo.chr9.4 revealed that AA genotypes comprised the Mdo.chr9.4- $\mathrm{R}_{0}$ dose 0 accessions, and $\mathrm{AB}$ genotypes comprised those of dose 1 and 2, suggesting that the association was overestimated for this SNP, since it neglected the allelic composition nature of $M d M Y B 1$. Thus, we excluded this SNP from the dataset and analyzed GWA again. A revised GWA analysis identified one association on unanchored chromosome (Fig. 3). The most significant marker was Mdo.chr9.4, scored as the dose of Mdo.chr9.4-R $\mathrm{R}_{0}$. Mdo.chr9.4 explained $52.0 \%$ of the phenotypic variation. To elucidate the dose effect of Mdo.chr9.4- $\mathrm{R}_{0}$, the relationship of the dose of Mdo.chr9.4- $\mathrm{R}_{0}$ and OCI was investigated using both 
Fig. 2 Distribution and correlation of over color and percent over color in the accession set (a) and fullsibs (b) based on a dose of Mdo.chr9.4- $\mathrm{R}_{0}$.

$* * * P<0.001$ by Pearson's correlation. Different letters indicate significant differences at $P<0.05$, as determined by Steel-Dwass tests
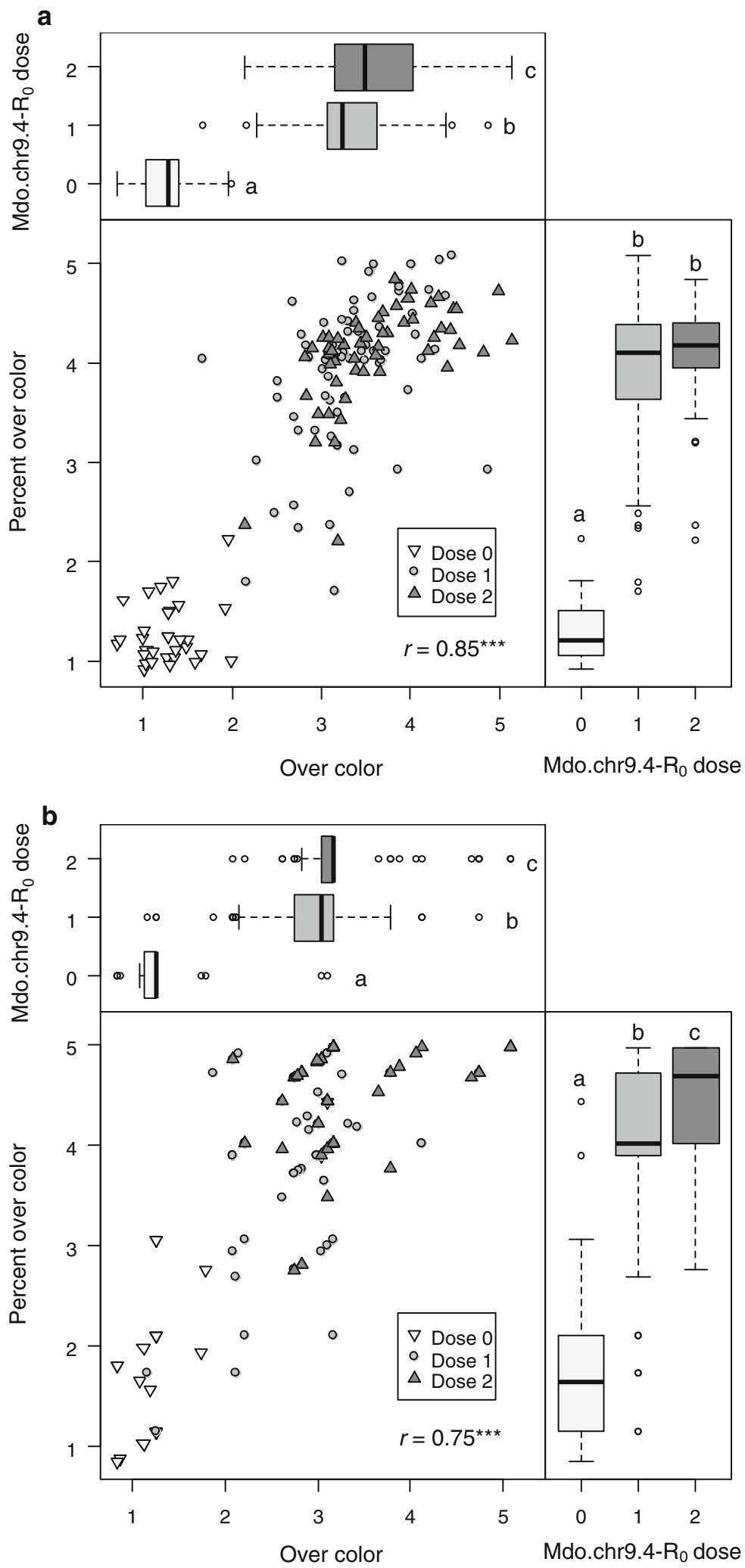


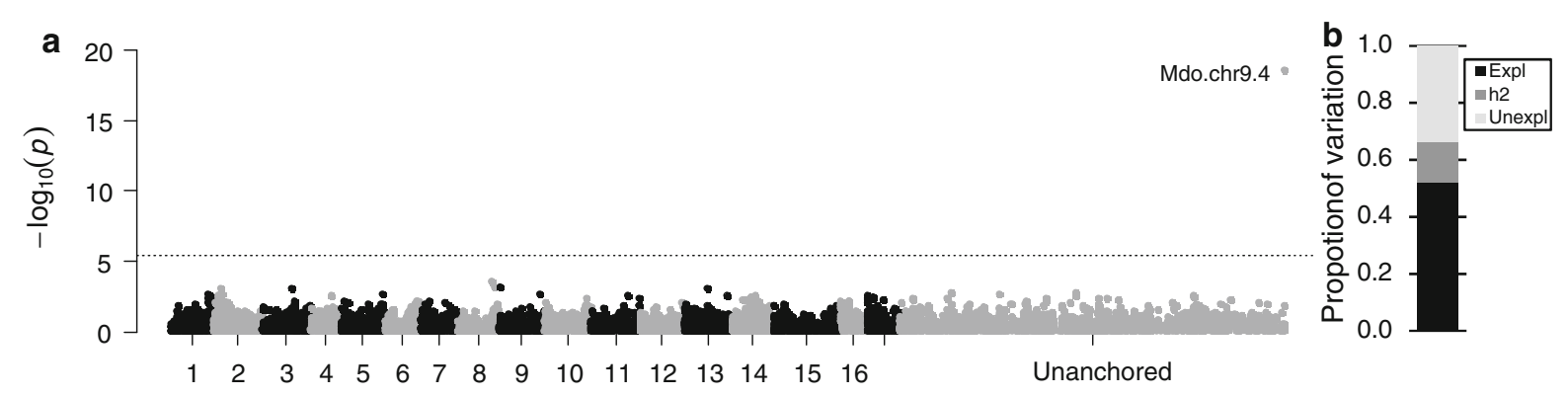

\section{Chromosome}

Fig. 3 Genome wide association analysis of overall color intensity in apples. A Manhattan plot of the $-\log _{10}(P)$ values of all the tested SNPs aligned according to Bianco et al. (2014), (a); variation distribution plot (b). Chromosomes are shown in two colors and are ordered from 1 to 17 and unanchored. "Unanchored" chromosome is a fictive one for placing the

AS and FSs. Significant differences in OCI were identified in all dose pairs $(0-1,0-2$, and 1-2) in the two datasets (Fig. 1). The comparisons within FS found that the dose $0-1$ and $0-2$ differences were always significant, whereas the dose 1-2 differences were significant in two ('Hatsuaki' $\times$ 'Megumi' and 'Sensyu' $\times$ 'Kotaro') out of the five FSs (Table 2). However, average, minimum, and maximum OCIs were all higher in dose 2 subgroups than in dose 1 in all the FSs. Quantitative effect calculation resulted in detection of semidominant effect of MdMYB1-1 for OCI in both AS and FSs, i.e., D/A were 0.74 and 0.56 in AS and FSs, respectively.

The GWAS for OC and POC identified one association for each (Online Resource 6). Mdo.chr9.4 explained $55.1 \%$ of the phenotypic variation and SNP_FB_0819307 (rs796058851, at MDC004836.412 chr9:30,440,192.30,463,669) explained $51.6 \%$ of the phenotypic variation and were the most significant for OC and POC, respectively. For the POC analysis step 0 that did not take account of the SNP cofactor in the model, Mdo.chr9.4 was the second most significant (data not shown). The $r^{2}$ between those markers was 0.84 , and the physical distance was approximately $500 \mathrm{~kb}$, according to the apple genome version 1 . Thus, we considered that this SNP reflected the association of MdMYB1 and skin color. However, SNP_FB_0819307 showed critical inconsistencies between genotype and phenotype in two accessions, i.e., 'Hida', a red cultivar, showed a null state of the red coloration allele and 'Gold Rush', a yellow cultivar, showed a heterozygous state of the red
SNPs anchored only to the "scaffold." The name of the significant marker is shown. The horizontal line indicates the genome-wide significance threshold, defined by the Bonferroni adjustment. Expl variation explained by significant marker, $h 2$ variation explained by genetic factors other than significant marker, Unexpl unexplained variation

coloration allele. These inconsistencies could have been caused by a recombination between MdMYB1 and SNP_FB_0819307 during meiosis. OC and POC differences for all Mdo.chr9.4- $\mathrm{R}_{0}$ dose pairs were significant in the two datasets, except for the dose 1-2 difference for POC in AS ( $P=0.53$; Fig. 2).

Validation for a marker detection system in the breeding program

We performed genotyping for Mdo.chr9.4 on AFs in order to demonstrate the validity of our marker detection system for breeding use (Table 3; Online Resource 7). The system comprised formatted nursing and crude DNA extraction from very young seedlings that resulted in an efficient and successful discrimination of the Mdo.chr9.4- $\mathrm{R}_{0}$ dose. In two of the five FSs, segregation distortion from the expected proportion was observed (Table 3).

\section{Discussion}

Allelic composition of Mdo.chr9.4 is associated with red skin color intensity

In the present study, we developed Mdo.chr9.4, which succeeded in MdMYBI allele discrimination (Table 1). We identified the only association at the bottom of chromosome 9 on which $M d M Y B 1$ was located (Fig. 3), confirming the role of MdMYB1 for apple fruit skin coloration. Moreover, we also 
developed an efficient genotyping system to apply on seedlings in breeding toward intensive red skin color (Tables 2, 3).

In previous studies, the DNA markers available for skin color selection showed inconsistencies between the phenotype and genotype, even in major cultivars, suggesting that they do not sufficiently capture allelic variations of $M d M Y B 1$ and were insufficient to distinguish the polymorphism or to be used in practical breeding. On the other hand, Mdo.chr9.4 captured allelic variations at least whether an allele has reddening ability, although it is also incomplete inventory of the MdMYB1 alleles. For example, a derived cleaved amplified polymorphic sequence (dCAPs) marker developed from the SNP located $-1664 \mathrm{bp}$ from the transcription start site of MdMYB1 identified the allele associated with MdMYB1-1 in 'Granny Smith,' a green skinned cultivar (Takos et al. 2006; Zhu et al. 2011). In order to solve this inconsistency, Zhu et al. (2011) proposed that the MdMYB1-4 dCAPS allele amplified the equivalent length to $M d M Y B 1-1$ but did not confer anthocyanin accumulation. We identified Mdo.chr9.4-Y -9 $_{\text {f }}$ from 'Granny Smith' that can be differentiated from reddening allele and may be associated with MdMYB1-4 (Tables 1, 4). Additionally, genotypes of 'Cripps' Pink' (MdMYB1-1/MdMYB1-3; Mdo.chr9. 4-R $\left.\mathrm{R}_{0} / \mathrm{Mdo} . \mathrm{chr} 9.4-\mathrm{Y}_{-15}\right)$ and 'Golden Delicious' (MdMYB1-2/MdMYB1-3; Mdo.chr9.4-Y ${ }_{-3}$ /Mdo.chr9. $4-Y_{-15}$ ) allow us to infer that MdMYB1-2 and MdMYB1-3 are associated with Mdo.chr9.4-Y -3 and Mdo.chr9.4-Y $\mathrm{Y}_{-15}$, respectively. Another example is that sequencing of $700 \mathrm{bp}$ promoter region around -1600 bp of MdMYB1 (Yuan et al. 2014) identified $M d M Y B 1-1$ in 'Indo,' which bears green with slightly red brushed fruit (Table 4; Supplementary Fig. S2). On the other hand, Mdo.chr9.4 identified homozygous state of Mdo.chr9.4-Y ${ }_{-3}$ in 'Indo,' being consistent with its skin color. This fact, additionally, also suggested that Mdo.chr9.4-Y $\mathrm{Y}_{-3}$ associated with several MdMYBI alleles. Detailed association between MdMYB1 and Mdo.chr9.4 should be confirmed by following study.

Chagné et al. (2016) proposed the SNP ss475879531 as a selective marker for MdMYB1; however, it is located $878 \mathrm{~kb}$ upstream of MdMYB1 according to the Apple genome version 1. A certain physical distance between the marker and gene of interest could raise a recombination between them, as we observed at SNP_FB_0819307. On the other hand, Mdo.chr9.4 was located only $8.3 \mathrm{~kb}$ away from $M d M Y B 1$, suggesting less risk of recombination than ss475879531 SNP. Consequently, Mdo.chr9.4 could currently be the best DNA marker to distinguish MdMYB1 polymorphism.

It is noteworthy that the OCI of dose 2 individuals was significantly greater than that of dose 1 individuals, which was however closer from dose 2 individuals than from dose 0 individuals, indicating a partially dominant effect of MdMYB1-1 (Fig. 1). These results indicated that $M d M Y B 1$ was the major and principal genetic factor, not only as a red skin color determinant, but also of the red intensity variation in apples. Thus, the selection of Mdo.chr9.4- $\mathrm{R}_{0}$ dose 2 individuals was expected to result in the selection of individuals with an intense red coloration. This finding is consistent with the observation of Chagné et al. (2016). To the best of our knowledge, the quantitative effect of the MdMYB1-1 allele on red skin color intensity was surely clarified for the first time. The quantitative effect of $M Y B$-related genes on anthocyanin accumulation was also reported in the berry skin color of grapevine (Azuma et al. 2008, 2011). Although a wide range of skin color was investigated, the expression level of MdMYB1 and skin color are positively correlated (Takos et al. 2006; Ban et al. 2007; Espley et al. 2007), suggesting that the different expression level among doses contribute to skin color intensity formation. To understand the common genetic and environmental control in anthocyanin accumulation in fruit, the mechanism behind these quantitative effects needs to be clarified.

Although fruit color is generally recognized as qualitative and dominantly inherited trait (Brown 2012), the OCI in our FSs was distributed continuously (Fig. 1b). Zhu et al. (2011) also observed a continuous distribution of skin color and incongruences of skin color and marker genotype in several individuals in two breeding populations. These observations match to breeder's insight that apple skin color is rather quantitative trait than qualitative. Because of a large contribution of MdMYB1 to phenotypic variation, simplified binary phenotyping approach taken in previous studies (Cheng et al. 1996; Takos et al. 2006) may have worked well. Continuous distribution of skin color may be originated from the accumulation of small-effect genetic factors which affect skin color even though a certain dose of Mdo.chr9.4 exists. These small-effect genetic factors 
Table 4 Relationship between MdMYB1 genotypes and Mdo.chr9.4 alleles in several accessions

\begin{tabular}{llllll}
\hline Accession & Gene/marker & Alleles & & $\begin{array}{l}\text { Polymorphism(s) used for } \\
\text { allele discrimination }\end{array}$ & Reference \\
\hline Cripps' Pink & MdMYB1 & 1 & 3 & -2000 to +4000 & Takos et al. (2006) \\
& Mdo.chr9.4 & $\mathrm{R}_{0}$ & $\mathrm{Y}_{-15}$ & +12363 & Present study \\
Golden Delicious & MdMYB1 & 2 & 3 & -2000 to +4000 & Takos et al. (2006) \\
& Mdo.chr9.4 & $\mathrm{Y}_{-3}$ & $\mathrm{Y}_{-15}$ & +12363 & Present study \\
Granny Smith & MdMYB1 & $2 / 3^{\mathrm{b}}$ & $4 \mathrm{a}^{\mathrm{c}}$ & -1661 & Zhu et al. (2011) \\
& Mdo.chr9.4 & $\mathrm{Y}_{-3}$ & $\mathrm{Y}_{-9}$ & +12363 & Present study \\
Indo & MdMYB1 & 1 & $4 \mathrm{~b}$ & -1400 to -700 & Yuan et al. (2014) \\
& Mdo.chr9.4 & $\mathrm{Y}_{-3}$ & $\mathrm{Y}_{-3}$ & +12363 & Present study \\
\hline
\end{tabular}

a Nucleotide position counted from the transcription start site of MdMYB1

b Undetermined by Zhu et al. (2011) but probably MdMYB1-2

c Zhu et al. (2011) and Yuan et al. (2014) gave identical name to suspicious different alleles, thus they are suffixed with the intent of better discrimination

are also likely to contribute to the OCI distribution within the same dose groups of Mdo.chr9.4- $\mathrm{R}_{0}$. However, it could be obvious that these small-effect genetic factors did not severely affect the fruit color because the proportion of individuals that showed an inconsistency was small (Fig. 1). The non-significant difference for OCI between dose 1 and 2 individuals in the three FSs may have been caused by the small number of investigated individuals and unprecise estimation of genotypic value due to the lower number of replications in the phenotypic assessment. Consequently, in the context of breeding, the selection of individuals with one or two doses of Mdo.chr9.4- $\mathrm{R}_{0}$, which was associated with the MdMYBI-1 allele coffering red skin coloration in fruits, allows us to choose those bearing red-skinned fruit. To obtain individuals with an intensive red skin color, it could be effective to select dose 2 individuals. Taken together, codominant marker, Mdo.chr9.4, along with AS and FSs enabled further discrimination of skin color within red skinned individuals, identifying that MdMYB1-1 dosage drives overall skin color intensity.

The high correlations between OC and POC (Fig. 2) suggested that the more intensive the red fruit, the broader the red-covered fruit surface while both traits were mainly controlled by MdMYB1. Additionally, individuals bearing well-colored fruit could be obtained even from the dose 1 subgroup by phenotypic selection. The non-significant difference for the POC between Mdo.chr9.4- $\mathrm{R}_{0}$ dose 1 and 2 individuals in AS was probably explained by the influence of selection for skin color in the breeding process. Our AS mainly consisted of modern Japanese elite apple cultivars that have been intensively selected for their red skin. The selection resulted in the elimination of apples showing moderate coloration in terms of POC, causing a different distribution compared to non-selected FSs (Fig. 1). Concerning the FS dataset that was not selected for skin color, the existence of low POC individuals in the dose 1 and 2 subgroups and the significant difference of POC between the dose 1 and 2 subgroups supported this hypothesis. These results suggested that, in addition to individuals with dose 2 , individuals with dose 1 could be favorable to breed new cultivars showing a high adaptability to a global warming climate in terms of red skin coloration.

It has recently been revealed that epigenetic regulation affects the expression of MdMYB1 and alters anthocyanin accumulation. Methylation in the MdMYB1 promoter region induced the yellow skinned bud sport in 'Gala' (El-Sharkawy et al. 2015). Demethylation also enhanced the expression of MdMYB1-2 and MdMYB1-3 and induced anthocyanin accumulation in 'Mutsu,' which was originally a yellow/green skinned cultivar (Bai et al. 2016). These results suggest that a causal mutation of skin reddening differences in MdMYB1 could underlie expression regulatory mechanisms located in the promoter region. A quantitative trait locus (QTL) for skin reddening located in the MdMYB1 region in 'Akane' (Kunihisa et al. 2014), an MdMYB1-1 and Mdo.chr9.4-R 
homozygous red skinned cultivar, suggests the existence of an unknown allelic variation, even in MdMYB1-1 from natural resources. These results imply the difficulty for developing a definitive DNA marker that can discriminate the variation of red intensity in detail without considering epigenetic aspects. It could be a future challenge to identify the causal polymorphism of $M d M Y B 1$ and to develop definitive marker(s) for red skin color selection.

\section{Marker detection system for use in breeding}

Our marker detection system successfully distinguished doses of Mdo.chr9.4- $\mathrm{R}_{0}$ within five FSs, despite the fact that two of them showed a distorted segregation (Table 3 ). This system was considered to be applicable to breeding for at least three reasons. The first is that our seedling nursing format which imitates a 96-well PCR plate can be adapted by most DNA marker detection methods, such as thermal cycler, automated DNA sequencer, and real-time PCR system. The second is that our assessment using early stage seedling saved time and space. It also allowed for an easy and intact replacement of Jiffy pots in order to remove unfavorable genotypes and seeds that did not germinate. The third is that our DNA extraction requires a single plate during PCR template preparation and the use of harmful chemicals such as liquid nitrogen and chloroform is unnecessary, thanks to the simple and crude extraction method developed by Thompson and Henry (1995). Using only single plates allows us to avoid any sample transfer from plate to plate that can cause cross-contamination. Moreover, it is cost-effective since the procedure does not need a kit provided by a commercial company. From our empirical view, the leaf tip collection from 96 individuals took $20 \mathrm{~min}$ at most and the subsequent steps took an additional $20 \mathrm{~min}$. It seems plausible that our template preparation procedure is easier than outsourcing (Baumgartner et al. 2016) and extraction using silica beads (Edge-Garza et al. 2014). We confirmed that genomic DNA prepared by our method could be used as the template for multiplex PCR (Moriya, unpublished), providing more value to the system when applied to MAS for multiple traits. However, we have not yet obtained firm result on how long the extracted DNA maintains its activity as a PCR template. Thus, at the moment, we recommend that it is used immediately after extraction. We believe that our system currently allows the earliest and most efficient MAS in apple breeding programs.

Acknowledgements We would like to thank the breeding sections of the following institutes for kindly providing plant materials of domestic cultivars; Akita Fruit Tree Experiment Station, Apple Research Institute of the Aomori Prefectural Industrial Technology Research Center, Fukushima Agricultural Technology Center, Gunma Agricultural Technology Center, Hokkaido Research Organization Agriculture Research Department, Ishikawa Agriculture and Forestry Research Center, Iwate Agricultural Research Center, Miyagi Prefectural Institute of Agriculture and Horticulture, Nagano Fruit Tree Experiment Station and Yamagata Prefectural Horticultural Experiment Station. We also thank Dr. Charles-Eric Durel (IRHS, INRA, France) for providing valuable suggestions for data interpretation. This study was supported by grants from the Ministry of Agriculture, Forestry, and Fisheries of Japan for "Genomics based Technology for Agricultural Improvement, NGB-2007”.

\section{Compliance with ethical standards}

Conflict of interest Authors declare that they have no conflict of interest.

\section{References}

Azuma A, Kobayashi S, Mitani N, Shiraishi M, Yamada M, Ueno T, Kono A, Yakushiji H, Koshita Y (2008) Genomic and genetic analysis of $M y b$-related genes that regulate anthocyanin biosynthesis in grape berry skin. Theor Appl Genet 117:1009-1019

Azuma A, Udo Y, Sato A, Mitani N, Kono A, Ban Y, Yakushiji H, Koshita Y, Kobayashi S (2011) Haplotype composition at the color locus is a major genetic determinant of skin color variation in Vitis $\times$ labruscana grapes. Theor Appl Genet 122:1427-1438

Bai S, Tuan PA, Saito T, Honda C, Hatsuyama Y, Ito A, Moriguchi $\mathrm{T}$ (2016) Epigenetic regulation of $M d M Y B 1$ is associated with paper bagging-induced red pigmentation of apples. Planta 244:573-586

Ban Y, Honda C, Hatsuyama Y, Igarashi M, Bessho H, Moriguchi T (2007) Isolation and functional analysis of a $M Y B$ transcription factor gene that is a key regulator for the development of red coloration in apple skin. Plant Cell Physiol 48:958-970

Baumgartner IO, Kellerhals M, Costa F, Dondini L, Pagliarani G, Gregori R, Tartarini S, Leumann L, Laurens F, Patocchi A (2016) Development of SNP-based assays for disease resistance and fruit quality traits in apple (Malus $\times$ domestica Borkh.) and validation in breeding pilot studies. Tree Genet Genomes 12:35

Bianco L, Cestero A, Sargent DJ, Banchi E, Derdak S, Di Gualdo M, Salvi S, Jansen J, Viola R, Gut I, Laurens F, Chagné D, velasco R, van de Weg E, Troggio M (2014) Development and validation of a $20 \mathrm{~K}$ single nucleotide 
polymorphism (SNP) whole genome genotyping aray for apple (Malus $\times$ domestica Borkh). PLoS ONE 9:e110377

Brown SK (2012) Apple. In: Badenes ML, Byrne DF (eds) Fruit breeding, handbook of plant breeding, vol 8. Springer, New York, pp 329-367

Chagné D, Kirk C, How N, Whitworth C, Fontic C, Reig G, Sawyer G, Rouse S, Poles L, Gardiner SE (2016) A functional genetic marker for apple red skin coloration across different environments. Tree Genet Genomes 12:1-9

Cheng FS, Weeden NF, Brown SK (1996) Identification of codominant RAPD markers tightly linked to fruit skin color in apple. Theor Appl Genet 93:222-227

Edge-Garza DA, Rowland TV, Haendiges S, Peace C (2014) A high-throughput and cost-efficient DNA extraction protocol for the tree fruit crops of apple, sweet cherry, and peach relying on silica beads during tissue sampling. Mol Breed 34:2225-2228

El-Sharkawy I, Liang D, Xu K (2015) Transcriptome analysis of an apple (Malus $\times$ domestica) yellow fruit somatic mutation identifies a gene network module highly associated with anthocyanin and epigenetic regulation. J Exp Bot 66:7359-7376

Espley RV, Hellens RP, Putterill J, Stevenson DE, Kutty-Amma S, Allan AC (2007) Red colouration in apple fruit is due to the activity of the MYB transcription factor, MdMYB10. Plant J 49:414-427

Espley RV, Brendolise C, Chagne D, Kutty-Amma S, Green S, Volz R, Putterill J, Schouten HJ, Gardiner SE, Hellens RP, Allan AC (2009) Multiple repeats of a promoter segment causes transcription factor autoregulation in red apples. Plant Cell 21:168-183

Iglesias I, Salvia J, Torguet L, Montserrat R (2005) The evaporative cooling effects of overtree microsprinkler irrigation on 'Mondial Gala' apples. Scientia Hort 103:267-287

Ju Z, Duan Y, Ju Z (1999) Effects of covering the orchard floor with reflecting films on pigment accumulation and fruit coloration in 'Fuji' apples. Sci Hortic 82:47-56

Jung S, Ficklin SP, Lee T, Cheng C-H, Blenda A, Zheng P, Yu J, Bombarely A, Cho I, Ru S, Evans K, Peace C, Abbott AG, Mueller LA, Olmstead MA, Main D (2014) The genome database for rosaceae (GDR): year 10 update. Nucleic Acids Res 42:D1237-D1244

Kumar S, Garrik DJ, Bink MCAM, Whitworth C, Chagne D, Volz RK (2013) Novel genomic approaches unravel genetic architecture of complex traits in apple. BMC Genom 14:393

Kunihisa M, Moriya S, Abe K, Okada K, Haji T, Hayashi T, Kim H, Nishitani C, Terakami S, Yamamoto T (2014) Identification of QTLs for fruit quality traits in Japanese apples: QTLs for early ripening are tightly related to preharvest fruit drop. Breed Sci 64:240-251

Kunihisa M, Moriya S, Abe K, Okada K, Haji T, Hayashi T, Kawahara Y, Itoh R, Itoh T, Katayose Y, Kanamori H, Toshimi M, Mori S, Sasaki H, Matsumoto T, Nishitani C, Yamamoto T (2016) Genomic dissection of a 'Fuji'apple cultivar: re-sequencing, SNP marker development, definition of haplotypes, and QTL detection. Breed Sci 66:499-515

Lin-Wang K, Bolitho K, Grafton K, Kortstee A, Karunairetnam S, McGhie TK, Espley RV, Hellens RP, Allan AC (2010) An R2R3 MYB transcription factor associated with regulation of the anthocyanin biosynthetic pathway in Rosaceae. BMC Plant Biol 10:50

Lin-Wang K, Micheletti D, Palmer J, Voltz R, Lozano L, Espley $\mathrm{R}$, Hellens RP, Chagné D, Rowan DD, Troggio M, Iglesias I, Allan AC (2011) High temperature reduces apple fruit colour via modulation of the anthocyanin regulatory complex. Plant Cell Environ 34:1176-1190

Lipka A, Tian F, Wang Q, Peiffer J, Li M, Bradbury PJ, Gore MA, Buckler ES, Zhang Z (2012) GAPIT: genome association and prediction integrated tool. Bioinformatics 28:2397-2399

Lozano L, Iglesias I, Micheletti D, Troggio M, Kumar S, Volz RK, Allan AC, Chagne D, Gardiner SE (2014) Feasibility of genome-wide association analysis using a small single nucleotide polymorphism panel in an apple breeding population segregating for fruit skin color. J Am Soc Hortic Sci 139:619-626

Migicovsky Z, Gardner KM, Money D, Sawler J, Bloom JS, Moffett P, Chao CT, Schwaninger H, Fazio G, Zhong GY, Myles S (2016) Genome to phenome mapping in apple using historical data. Plant Genome. doi:10.3835/ plantgenome2015.11.0113

Moriya S, Okada K, Haji T, Yamamoto T, Abe K (2012) Fine mapping of $\mathrm{Co}$, a gene controlling columnar growth habit located on apple (Malus x domestica Borkh.) linkage group 10. Plant Breed 131:641-647

Moriya S, Kunihisa M, Okada K, Iwanami H, Iwata H, Minamikawa M, Katayose Y, Matsumoto T, Mori S, Sasaki H, Matsumoto T, Nishitani C, Terakami S, Yamamoto T, Abe K (2016) Identification of QTLs for flesh mealiness in apple (Malus $\times$ domestica Borkh.). Hortic J. doi:10.2503/ hortj.MI-156

R Core Team (2016) R: a language and environment for statistical computing. R Foundation for Statistical Computing, Vienna. https://www.R-project.org/

Segura V, Vilhjalmsson BJ, Platt A, Korte A, Seren U, Long Q, Nordborg M (2012) An efficient multi-locus mixed-model approach for genome-wide association studies in structured populations. Nat Genet 44:825-830

Takos AM, Jaffe FW, Jacob SR, Bogs J, Robinson SP, Walker AR (2006) Light-induced expression of a $M Y B$ gene regulates anthocyanin biosynthesis in red apples. Plant Physiol 142:1216-1232

Thomson D, Henry R (1995) Single-step protocol for preparation of plant-tissue for analysis by PCR. Biotechniques 19:394-400

Velasco R, Zharkikh A, Affourtit J, Dhingra A, Cestaro A, Kalyanaraman A, Fontana P, Bhatnagar SK, Troggio M, Pruss D, Salvi S, Pindo M, Baldi P, Castelletti S, Cavaiuolo M, Coppola G, Costa F, Cova V, Dal Ri A, Goremykin V, Komjanc M, Longhi S, Magnago P, Malacarne G, Malnoy M, Micheletti D, Moretto M, Perazzolli M, Si-Ammour A, Vezzulli S, Zini E, Eldredge G, Fitzgerald LM, Gutin N, Lanchbury J, Macalma T, Mitchell JT, Reid J, Wardell B, Kodira C, Chen Z, Desany B, Niazi F, Palmer M, Koepke T, Jiwan D, Schaeffer S, Krishnan V, Wu C, Chu VT, King ST, Vick J, Tao Q, Mraz A, Stormo A, Stormo K, Bogden R, Ederle D, Stella A, Vecchietti A, Kater MM, Masiero S, Lasserre P, Lespinasse Y, Allan AC, Bus V, Chagne D, Crowhurst RN, Gleave AP, Lavezzo E, Fawcett JA, Proost S, Rouze P, Sterck L, Toppo S, Lazzari B, Hellens RP, 
Durel CE, Gutin A, Bumgarner RE, Gardiner SE, Skolnick M, Egholm M, Van de Peer Y, Salamini F, Viola R (2010) The genome of the domesticated apple (Malus $\mathrm{x}$ domestica Borkh.). Nat Genet 42:833-839

You FM, Huo N, Gu YQ, Luo M-C, Ma Y, Hane D, Lazo GR, Dvorak J, Anderson OD (2008) BatchPrimer3: a high throughput web application for PCR and sequencing primer design. BMC Bioinform 9:253

Yu J, Pressoir G, Briggs WH, Vroh Bi I, Yamasaki M, Doebley JF, McMullen MD, Gaut BS, Nielsen DM, Holland JB, Kresovich S, Buckler ES (2006) A unified mixed-model method for association mapping that accounts for multiple levels of relatedness. Nat Genet 38:203-208
Yuan KJ, Wang CJ, Wang JH, Xin L, Zhou GF, Li LG, Shen GN (2014) Analysis of the MdMYB1 gene sequence and development of new molecular markers related to apple skin color and fruit-bearing traits. Mol Genet Genom 289:1257-1265

Zhang XJ, Wang LX, Chen XX, Liu YL, Meng R, Wang YJ, Zhao ZY (2014) A and MdMYB1 allele-specific markers controlling apple (Malus $\times$ domestica Borkh.) skin color and suitability for marker-assisted selection. Genet Mol Res 13:9103-9114

Zhu YM, Evans K, Peace C (2011) Utility testing of an apple skin color MdMYB1 marker in two progenies. Mol Breed 27:525-532 